\title{
SalA Attenuates Hypoxia-Induced Endothelial Endoplasmic Reticulum Stress and Apoptosis via Down-Regulation of VLDL Receptor Expression
}

\author{
Ping Xie ${ }^{a}$ Yingchun Duan ${ }^{b}$ Xianzhi Guo Lina Hua Minghua Yua \\ a Department of Oncology, ${ }^{b}$ Department of Obstetrics and Gynecology, Shanghai Pudong Hospital, \\ Fudan University, Shanghai
}

\section{Key Words}

Salvianolic acid A - Hypoxia - VLDL receptor - Endothelial endoplasmic reticulum stress • Apoptosis

\begin{abstract}
Background: Salvianolic acid A (SalA) has been shown to display robust protection against endothelial injury. VLDL receptor (VLDLr) is expressed at high levels in the endothelial cells. However its endothelial biological function has not been completely elucidated. Here, we investigated molecular effects of SalA on endothelial VLDLr expression, ER stress, and apoptosis under hypoxia condition. Methods: Human umbilical vein endothelial cells (HUVECs) pretreated with SalA were subjected to hypoxia stimulation. Endothelial ER stress and apoptosis were examined. The mRNA levels were tested by real-time RT-PCR, and the protein levels were determined by immunoblot analysis. Results: Pretreatment of HUVECs with SalA markedly attenuated hypoxia-induced endothelial ER stress and apoptosis. Hypoxia resulted in enhancement of VLDLr expression, which was effectively inhibited by SalA pretreatment. Furthermore, luciferase reporter gene assays indicated that SalA inhibited vldlr gene promoter activity, and ChIP assays showed that hypoxia increase the recruitment of HIF-1 $\alpha$ to the vldlr gene promoter, and this process was hampered markedly by pretreatment of SalA. Finally, overexpression of VLDLr abolished SalA-mediated protection of endothelial cells from ER stress and apoptosis. Knockdown of VLDLr mimicked SalA protective effect. Conclusion: These results for the first time demonstrate that SalA protects against hypoxia-induced endothelial ER stress and apoptosis through inhibiting recruitment of HIF-1 $\alpha$ to vldlr gene promoter and thus suppressing VLDLr expression.
\end{abstract}


Xie et al.: SalA Attenuates Hypoxia-Induced Endothelial ER Stress

\section{Introduction}

The vascular endothelium consisting of a single cell layer lining all vessels, acts as a physiological barrier and plays a crucial role in maintaining the vascular homeostasis. The endoplasmic reticulum (ER) is a highly dynamic organelle almost present in all eukaryotic cells. Multiple factors both inside the cell and in the ER microenvironment influence ER functions, resulting in ER stress and thus impairing protein folding in the lumen of the ER. The disturbed protein folding process then leads to the accumulation of unfolded or misfolded proteins in the ER lumen, which then directs to the activation of a special cellular process called the unfolded protein response (UPR). Unreversed ER stress undoubtedly triggers cellular death programme. The ER is now recognized as an important organelle in deciding cell life and death. More recently, compelling evidence indicated a crucial role of disturbed ER functions in endothelial pathology and even cardiovascular disease, such as ischemic vascular disorder, neovascularization, and atherosclerosis [1-3].

The very-low-density-lipoprotein (VLDL) receptor (VLDLr) is a transmembrane lipoprotein acceptor of the low-density-lipoprotein (LDL) receptor family. VLDLr is expression abundantly in fatty acid tissues due to their high level of triglycerides, VLDLr's primary ligand. These other VLDLr-present tissues include those of the heart, skeletal muscle, and adipose layer. In addition, the receptor is also found in endothelial cells. Beyond the function of VLDLr as a peripheral lipoprotein receptor, the possibilities of its biological function have been extended to the process of signal transduction, angiogenesis, and even tumor growth [4]. Recently, some study indicates a modulatory role of VLDLr in the endothelial biological processes except lipoprotein metabolism. VLDLr was involved in the activation of retinal vascular endothelial cells and promotion of angiogenesis [5, 6]. Further, fibrin-dependent transendothelial migration of leukocytes was found to be associated with VLDLr [7]. VLDL receptor was mediated in microvascular endothelial cells to inhibit cell division [8]. More or less, these findings showed that a close relationship may be existed between VLDLr and endothelial pathological events. More recently, we found that hypoxia-induced VLDLr expression triggered endothelial ER stress and apoptosis.

S. miltiorrhiza, commonly named "Danshen", is a valuable medicinal plant used in China for thousand years to alleviate some significant symptoms of cardiovascular disease [9]. It is also an important ingredient of compound danshen dripping pills which have been passed phase II human clinical trial of U.S. Food and Drug Administration (FDA). Salvianolic acid A (SalA), is one of the main active, water-soluble components in S. miltiorrhiza. Previous studies have indicated beneficial effects of SalA on resistance to oxidative stress, platelet aggregation, ischemia, and hepatocirrhosis [10]. In the present study, we, for the first time, provide evidence for the protective effect of SalA on hypoxia-induced endothelial ER stress and apoptosis through down-regulation of VLDLr expression in human umbilical endothelial cells (HUVECs).

\section{Materials and Methods}

\section{Chemicals and reagents}

Anti-VLDL-receptor (VLDLr) antibody was obtained from R\&R Systems, and anti-cleaved caspase-3, anti-Bax, anti-Bcl-2, anti- $\beta$-actin and HRP-conjugated secondary antibodies were from Cell Signaling Technology. Anti-phospho-PERK, anti-PERK, anti-GRP78, and anti-CHOP were purchased from Santa Cruz Biotechnology. All other chemicals were from Sigma.

Cell culture and transfection of adenovirus and siRNA

Human umbilical endothelial cells (HUVECs) (Sciencell) were cultured in endothelial cell medium (ECM) supplemented with endothelial cell growth supplement (ECGS), 5\% fetal bovine serum (FBS), and penicillin/streptomycin (P/S) solution (Sciencell) in an incubator with $37^{\circ} \mathrm{C}, 5 \% \mathrm{CO}_{2}$. For experiments, cells of passage no. 4-6 were grown until confluence. Recombinant adenoviruses expression VLDLr or control 
viruses were constructed with the Ad-Easy system and cultures were routinely infected at a multiplicity of infection of five with an infective efficiency up to 80\%. The VLDL receptor (VLDLr) siRNA and nontargeting siRNA used in our study were designed by and purchased from Dharmacon. HUVECs were transfected with $50 \mathrm{nmol} / \mathrm{L}$ siRNA with DharmaFECT1 as described previously [14]. The nontargeting siRNA was used as transfection control for non-sequence-specific effects of the transfected siRNAs.

\section{Cellular hypoxia treatment}

For experimental hypoxia, cells were incubated with the serum-free medium and placed in an airtight humidified chamber with $37^{\circ} \mathrm{C}, 5 \% \mathrm{CO}_{2}$, and $95 \% \mathrm{~N}_{2}$. The matched normoxia control cells were cultured in a humidified incubator with $37^{\circ} \mathrm{C}, 5 \% \mathrm{CO}_{2}$, and $21 \% \mathrm{O}_{2}$.

\section{Promoter constructs and luciferase reporter gene assay}

The promoter fragments of vldlr were PCR-amplified from gemonic DNA and cloned into a luciferase vector at the Kpn1 and Bgl II site (pGL3; Promega, Madison, WI). The primers used for PCR amplification were as follows: 5'-GAGGGTACCAGTCGAGCCCCT-3' (sense); 5'-CTCAGATCTCATGCTGCCCGC-3' (antisense). HUVECs were seeded in 96-well plates and transfected with $100 \mathrm{ng}$ of luciferase vector (pGL3 constructs), and $25 \mathrm{ng}$ of Renilla vector (pRLTK) using Lipofectamine 2000 (Invitrogen). Twenty-four hours later, cells were subjected to hypoxia with or without SalA pretreatment and then lysed. The ratios between firefly luciferase and Renilla luciferase activity were measured with a dual-luciferase assay (Promega).

\section{Chromatin Immunoprecipitation Assay}

The chromatin immunoprecipitation assay (ChIP) was performed as described previously [11,12]. In brief, HUVECs were cross-linked with $1 \%$ formaldehyde at $37^{\circ} \mathrm{C}$ for $10 \mathrm{~min}$ at room temperature. The cell lysate was sonicated at $30 \%$ output on ice four times with 15-s intervals and pre-cleared with protein Sepharose 4B (Amersham Biosciences). The lysate was precipitated with the primary antibodies to HIF$1 \alpha(4 \mathrm{mg})$ overnight at $4^{\circ} \mathrm{C}$. The DNA was purified using the QIA-AMP DNA purification system (Qiagen). PCR products were detected, using primers specific for human VLDLr (386/524), forward primer: 5'-CCAACCAAGCCCTCCAGCAA-3', reverse primer: 5'-ATCAGTGGCACCCTCCTCCC-3'. Equal input DNA control was assessed and anti-Flag antibody (Sigma) was served as the negative control.

\section{Flow cytotmetry assay}

Cell apoptosis was analyzed by annexin V-FITC / propidium iodide (PI) staining according to the manufacturer's instructions (Invitrogen, USA). Briefly, cells were rinsed with ice-cold PBS and resuspended at a final concentration of $2-5 \times 10^{5} / \mathrm{ml}$ in $250 \mu$ l binding buffer. Five microliters of annexin V-FITC stock solution was added to the cells and rinsed for 3 minutes at $4^{\circ} \mathrm{C}$. Then $10 \mu \mathrm{l} \mathrm{PI}(20 \mu \mathrm{g} / \mathrm{ml})$ was added and incubated in the dark at room temperature for $10 \mathrm{~min}$. Cells were analyzed by flow cytometry (FACS Calibur, BD Biosciences, USA) equipped with Cell Quest software. For each sample, approximately $1 \times 10^{4}$ cells were analyzed.

\section{Quantitative real-time RT-PCR analysis}

Total RNA was purified from HUVECs with Trizol (Invitrogen). Realtime PCR was performed using Bio-RAD iQ5 Multicolor Real-Time PCR Detection System with SYBR Green as fluorescent and ROX (Takara) as reference dyes as described previously [13]. The specific primers used were as follows: human VLDLr: 5'-GTGGAAAATGTGATGGGGATG-3' (forward), 5'-CCATTGTTGCACACGAAGTC-3' (reverse); human GRP78: 5'-GCCGCAGGCGCTGGAAAGAT-3' (forward), 5'-GCCGATGAGTCGCTTGGCGT-3' (reverse); human CHOP: 5'-GGTGGCAGCGACAGAGCCAA-3' (forward), 5'-CATGCGTTGCTTGCCAGCCC-3' (reverse); human GAPDH: 5'-GACCCCTTCATTGACCTC-3' (forward), 5'-GCTAAGCAGTTGGTGGTG-3' (reverse)..

\section{Immunoblot analysis}

Immunoblotting was performed according to the previous protocols [14]. Total proteins were extracted from HUVECs using cell lysis buffer. Protein (40-60 ug) from each sample were separated by SDS-PAGE, transferred to nitrocellulose membranes, and probed with primary antibodies and then with horseradish peroxidase-conjugated secondary antibodies. The enhanced chemiluminescence signal was quantified using a densitometry program (Gel-pro 4.5 Analyzer, Media Cybernetics). To quantify the protein signal, we 
subtracted background, normalized the value to $\beta$-actin. As for the phospho-specific protein, we normalized the signal to the amount of total target protein and $\beta$-actin. The data of each group were expressed as a fold change of the control.

\section{Statistical analysis}

Each experiment was performed at least in triplicate. Data were presented as mean+SEM. Differences between groups were evaluated for statistical significance using Student's $t$ or ANOVA tests. In each case, significance was defined as $P<0.05$.

\section{Results}

SalA inhibits hypoxia-induced endothelial apoptosis

To investigate the biological role of SalA in hypoxia-induced endothelial dysfunctions, HUVECs were pretreated with SalA at a concentration of 50,100, or 200ng/L and subjected to hypoxia for $8 \mathrm{~h}$. As shown in Figure 1A, treatment of cells with hypoxia resulted in endothelial apoptosis, which was significantly suppressed by pretreatment of cells with SalA. Similarly, SalA markedly inhibited hypoxia-induced enhancement of protein levels of cleaved caspase-3 and pro-apoptotic Bax (Fig. 1B). In contrast, suppressed expression of anti-apoptotic Bcl2 was partly reversed by SalA at the concentration of 100 or 200ng/L (Fig. 1B). These data indicate a protective role of SalA in hypoxia-induced endothelial apoptosis.

\section{SalA attenuates hypoxia-induced endothelial ER stress}

Hypoxia is known to trigger ER stress, and recent evidence indicated that ER stress played an important role in the progression of cardiovascular disease $[3,15]$. We therefore investigated whether SalA might attenuate hypoxia-induced endothelial ER stress. Experimentally induced hypoxia in endothelial cells triggered elevated mRNA levels of Bip and CHOP, which were significantly alleviated by pretreatment of SalA at concentrations of 100 and 200ng/L (Fig. 2A). In agreement with the transcript levels, hypoxia-enhanced protein expression of Bip and CHOP were also inhibited by pretreatment of SalA (Fig. 1B). In addition, we observed that phosphoralylation of PERK displayed similar alterations with Bip and CHOP (Fig. 1B). These results suggest that SalA might attenuate hypoxia-induced ER stress in endothelial cells.

\section{SalA inhibits Hypoxia-enhanced expression of VLDLr}

In our previous study, we found that oxidative stress promoted expression of VLDLr which was involved in ER stress and apoptosis $[16,17]$. We therefore determine whether SalA affect the VLDLr expression. As shown in Figure 3A and B, hypoxia induced marked increase in mRNA and protein levels of VLDLr. Interestingly, pretreatment of SalA at concentrations of 100 and 200ng/L sufficiently abolished hypoxia-induced VLDLr expression (Fig. 3A and B). These results indicate that SalA might serve as an effective suppressor of VLDLr expression.

\section{SalA inhibits HIF-1 $\alpha$ binding to vldlr gene promoter}

In our previous study, binding of HIF- $1 \alpha$ to hypoxia-responsive element (HRE) in vldlr gene promoter (between -161 and -158 bp) triggered VLDLr expression [17]. Therefore, we examined the effect of SalA on the HIF-1 $\alpha$-dependentVLDLr expression. SalA pretreatment did not led to obvious alteration of HIF-1 $\alpha$ protein expression (Fig. 4A). However, SalA effectively inhibited vldlr gene promoter activity at the concentrations of 100 and 200ng/L (Fig. 4B). Thus, we further detected whether the binding of HIF-1 $\alpha$ to vldlr gene promoter was changed by SalA pretreatment. ChIP assays displayed that hypoxia increase the recruitment of HIF- $1 \alpha$ to the vldlr gene promoter, and this process was hampered markedly by pretreatment of SalA at the concentrations of 100 and 200ng/L (Fig. 4C). Collectively, SalA might inhibit HIF-1 $\alpha$ binding to vldlr gene promoter, leading to suppressed VLDLr expression.

\section{KARGER}




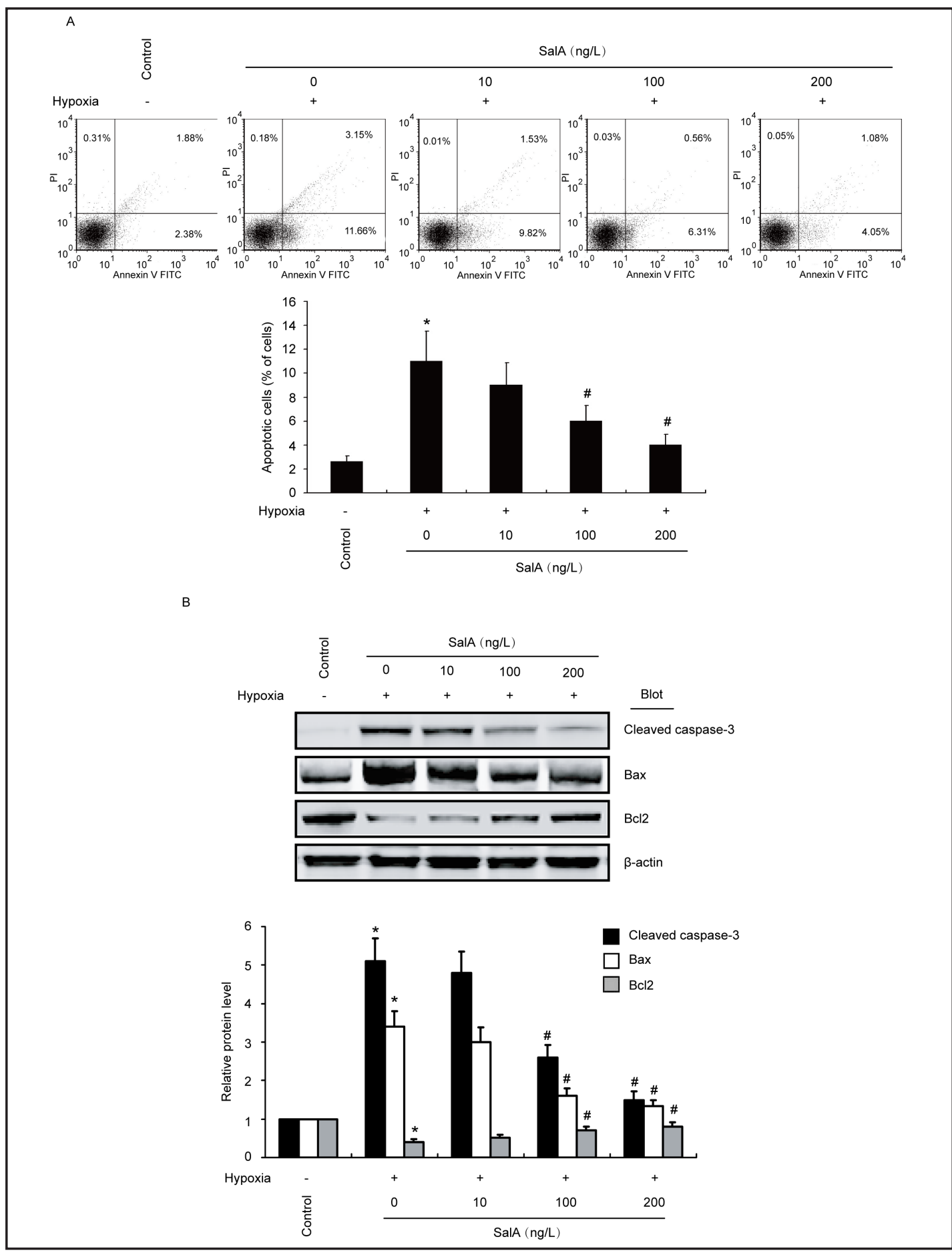

Fig. 1. SalA inhibits hypoxia-induced endothelial apoptosis. HUVECs were pretreated with SalA for 30 min in various concentrations, and then subjected to hypoxia for $8 \mathrm{~h}$. (A) The cell apoptosis was measured by flow cytometry analysis as described in Materials and Methods. Representative photos of different groups were shown. The apoptotic cells was quantified $(n=4$, mean \pm SEM). The values represent the mean percentages of apoptotic cells in different groups. ${ }^{*} P<0.05$ vs. control cells; ${ }^{\#} P<0.05$ vs. non-SalA-treated hypoxia cells. (B) The protein levels of cleaved caspase-3, $\mathrm{Bax}, \mathrm{Bcl} 2$, and $\beta$-actin were detected by immunoblot analysis using indicated antibodies. A representative blot (top) was shown ( $\mathrm{n}=4)$. Quantitative analysis of cleaved caspase-3, Bax, and Bcl2 (bottom) were expressed as fold increase above control group ( $\mathrm{n}=4$, mean $\pm \mathrm{SEM}$ ). ${ }^{*} P<0.05$ vs. control cells; ${ }^{\#} P<0.05$ vs. non-SalA-treated hypoxia cells. 


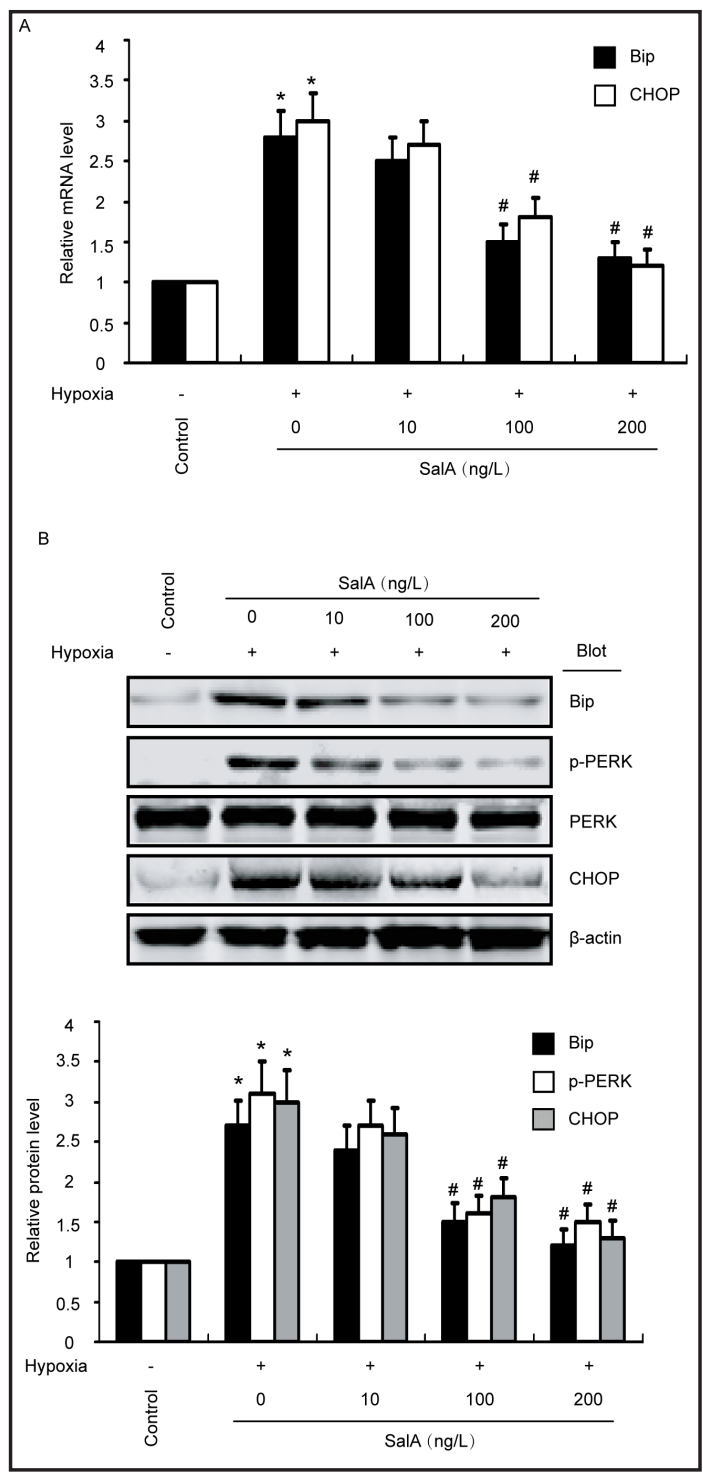

Fig. 2. SalA inhibits hypoxia-induced endothelial ER stress. HUVECs were pretreated with SalA for $30 \mathrm{~min}$ in various concentrations, and then subjected to hypoxia for $8 \mathrm{~h}$. (A) The relative expression levels of Bip and CHOP were detected by q-PT-PCR $(n=6$, mean+SEM). ${ }^{*} P<0.05$ vs. control cells; ${ }^{*} P<0.05$ vs. non-SalA-treated hypoxia cells. (B) The protein levels of Bip, p-PERK, PERK, CHOP, and $\beta$-actin were detected by immunoblot analysis using indicated antibodies. A representative blot (top) was shown ( $n=5)$. Quantitative analysis of Bip, p-PERK, and CHOP (bottom) were expressed as fold increase above control group ( $\mathrm{n}=4$, mean \pm SEM). ${ }^{*} P<0.05$ vs. control cells; ${ }^{*} P<0.05$ vs. non-SalA-treated hypoxia cells.

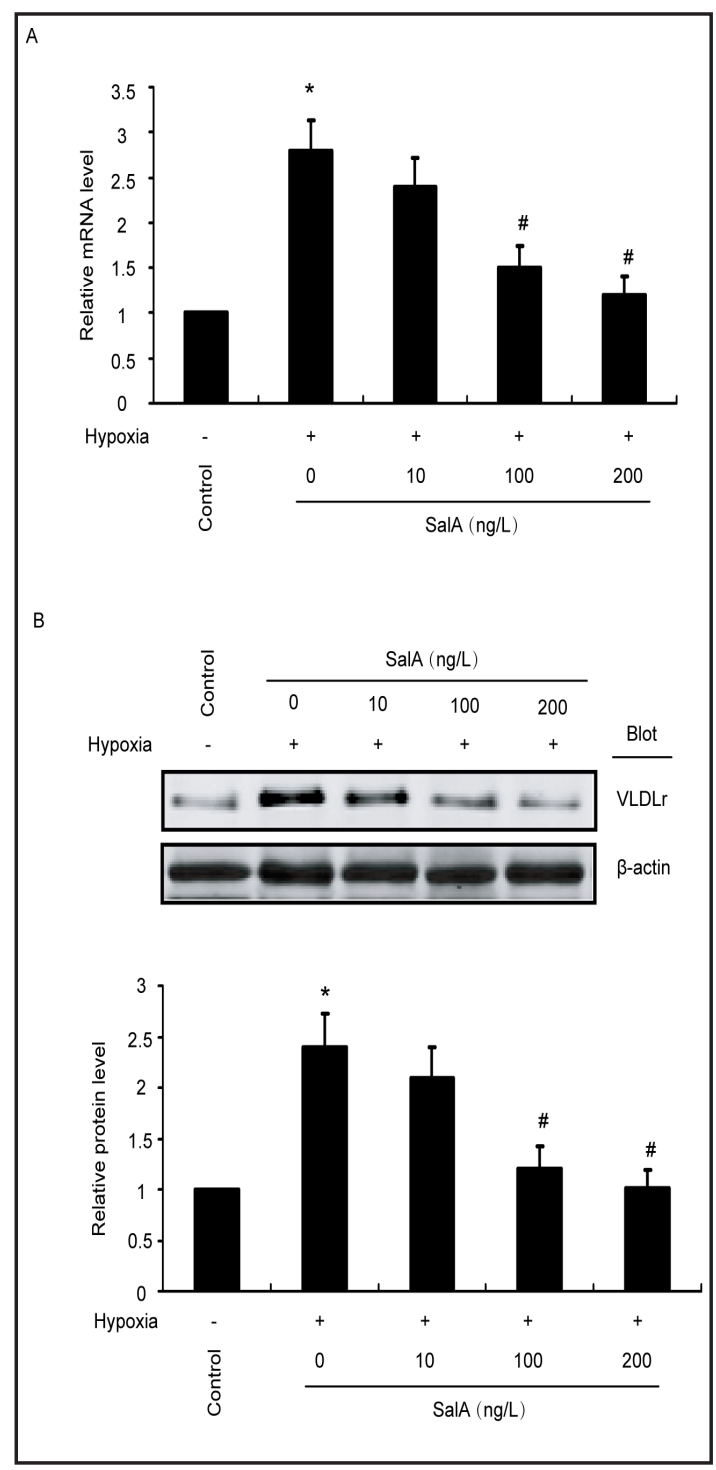

Fig. 3. SalA inhibits hypoxia-induced expression of VLDLr. HUVECs were pretreated with SalA for $30 \mathrm{~min}$ in various concentrations, and then subjected to hypoxia for 8h. (A) The VLDL receptor mRNA levels in each group were detected by quantitative real-time RT-PCR ( $n=5$, mean \pm SEM). ${ }^{*} P<0.05$ vs. control cells; ${ }^{\#} P<0.05$ vs. non-SalA-treated hypoxia cells. (B) The protein levels of VLDLr and $\beta$-actin were detected by immunoblot analysis using indicated antibodies. A representative blot (top) was shown ( $n=4)$. Quantitative analysis of VLDLr (bottom) were expressed as fold increase above control group $(n=4$, mean \pm SEM). ${ }^{*} P<0.05$ vs. untreated control cells; ${ }^{\#} P<0.05$ vs. non-SalA-treated IS/RP cells.

\section{VLDLr overexpression abolishes SalA-mediated protection of endothelial apoptosis}

To determine whether SalA protect endothelial cells form apoptosis through reducing VLDLr expression, we transfected cells with adenovirus to get cellular overexpression of 
Fig. 4. SalA supresses HIF-1 $\alpha$ binding to vldlr gene promoter. HUVECs were pretreated with SalA for $30 \mathrm{~min}$ in various concentrations, and then subjected to hypoxia for $8 \mathrm{~h}$. (A) The protein levels of HIF- $1 \alpha$ and $\beta$-actin were detected by immunoblot analysis using indicated antibodies. A representative blot was shown $(n=4)$. (B) The vldlr gene promoter activity was messured by luciferase reporter gene assay as described in Materials and Methods ( $\mathrm{n}=4$, mean \pm SEM). ${ }^{*} P<0.05$ vs. untreated control cells; ${ }^{\#} P<0.05$ vs. non-SalA-treated hypoxia cells. (C) Cells were analyzed using the ChIP assay with antibodies to HIF- $1 \alpha$ (see Materials and Methods). Figure is representative of three separate experiments.

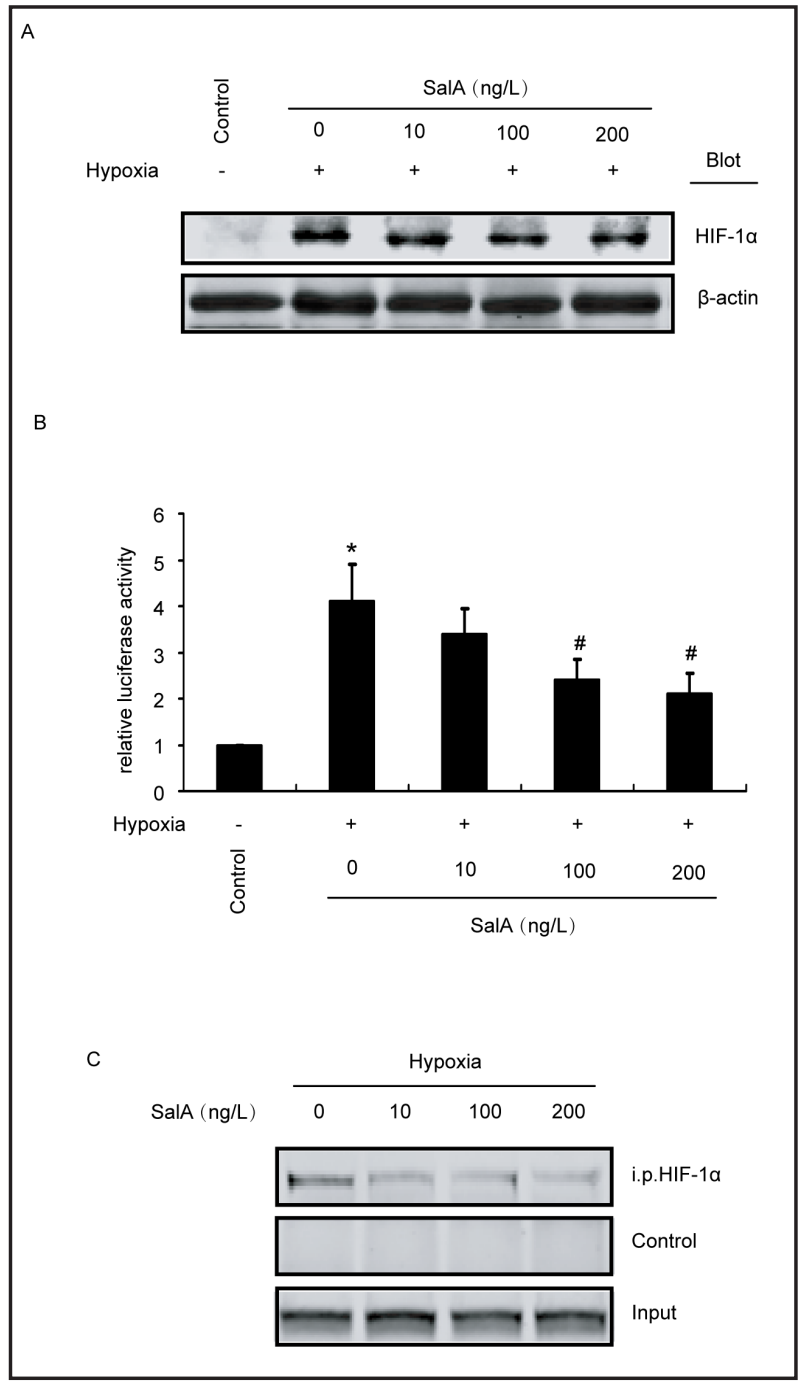

VLDLr. We showed that VLDLr overexpression completely abolished the protective role of SalA in endothelial apoptosis (Fig. 5A). Moreover, protein levels of cleaved caspase-3 and proapoptotic Bax was down-regulated after the SalA pretreatment, and this phenomenon was greatly reversed by VLDLr overexpression (Fig. 5B). Meanwhile, the elevated protein level of anti-apoptotic Bcl2 by SalA pretreatment was also disturbed after VLDLr overexpression (Fig. 5B). Furthermore, silencing of VLDLr mimicked the protective actions of SalA (Fig. 5B). Together, our data thus show that VLDLr might be an essential mediator through which SalA protects endothelial cells from apoptosis.

\section{VLDLr overexpression inhibits the suppression of ER stress mediated by SalA}

We further detect whether VLDLr overexpression affect the suppression of ER stress induced by SalA. As shown in Figure 6A, as a consequence of VLDLr overexpression, SalAsuppressed mRNA levels of Bip and CHOP were significantly reversed. In agreement with transcript levels, similar alterations were observed with regard to the protein levels of Bip, phosphor-PERK, and CHOP, manifested by decreased levels after SalA pretreatment and reversed levels after VLDLr overexpression despite SalA pretreatment (Fig. 6B). Moreover, the phenomenon of silencing of VLDLr was similar to that of SalA pretreatment (Fig. 6C). Therefore, these results undoubtedly indicate that VLDLr might be a crucial factor involving in suppression of ER stress induced by SalA. 
Fig. 5. VLDLr overexpression impaired protective role of SalA in endothelial apoptosis. (A) HUVECs were transfected with Ad-Control or Ad-VLDLr. After $48 \mathrm{~h}$, cells were pretreated with or without $100 \mathrm{ng} / \mathrm{L}$ SalA for 30 min, and then subjected to hypoxia for $8 \mathrm{~h}$. The cell apoptosis was measured by flow cytometry analysis and quantitative analysis of apoptotic cells was shown $(n=4$, means \pm SE). The values represent the mean percentages of apoptotic cells in different groups. $\left(\mathrm{n}=5\right.$, mean \pm SEM). ${ }^{*} P<0.05$ vs. non-SalA-treated hypoxia cells $(-) ;{ }^{\# \#} P<0.01$ vs. SalA-treated and Ad-Control-transfected cells. (B) Cells were treated as in A. The protein levels of VLDLr, cleaved caspase- 3 , $\mathrm{Bax}, \mathrm{Bcl} 2$, and $\beta$-actin were detected by immunoblot analysis using indicated antibodies. A representative blot was shown $(n=4)$. (C) Cells were transfected with nontargeting SiRNA (siNT) or VLDLr siRNA (siVLDLr). After 48h, cells were pretreated with or without 100ng/L SalA for $30 \mathrm{~min}$, and then subjected to hypoxia for $8 \mathrm{~h}$. The protein levels of VLDLr, cleaved caspase-3, Bax, Bcl2, and $\beta$-actin were detected by immunoblot analysis. A representative blot was shown $(n=3)$.

\section{Discussion}

In the present study, we provided evidence for the first time that SalA, a potential drug component from a medicinal plant, attenuated hypoxia-induced endothelial ER stress and apoptosis. The inhibited ER stress and apoptosis were due to the decreased VLDLr expression mediated by the events that SalA inhibited HIF- $1 \alpha$ binding to vldlr gene promoter, therefore leading to reduced gene promoter activity. Thus, our results provide a novel mechanism that could contribute to understand the protective action of SalA against hypoxia-indced ER stress and apoptosis in endothelial cells.

The vascular endothelium is a semi-selective diffusion barrier between the blood plasma and interstitial tissue. Besides its important function of regulating blood coagulation, the endothelium has been shown to play an essential role in the inflammatory processes, development of atherosclerotic lesions, and regulation of blood pressure. The endoplasmic reticulum (ER) is a complex membranous framework found in all eukaryotic cells [18]. The 
Fig. 6. VLDLr overexpression supressed protective role of SalA in endothelial ER stress. (A) HUVECs were transfected with Ad-Control or Ad-VLDLr. After $48 \mathrm{~h}$, cells were pretreated with or without $100 \mathrm{ng} / \mathrm{L}$ SalA for $30 \mathrm{~min}$, and then subjected to hypoxia for $8 \mathrm{~h}$. The relative expression levels of Bip and CHOP were detected by q-PT-PCR $(n=5$, mean+SEM). ${ }^{*} P<0.05$ vs. non-SalA-treated hypoxia cells ( - ; ${ }^{\#} P<0.05$ vs. SalA-treated and Ad-Control-transfected cells. (B) Cells were treated as in $\mathrm{A}$. The protein levels of Bip, p-PERK, PERK, CHOP, and $\beta$-actin were detected by immunoblot analysis using indicated antibodies. A representative blot was shown $(n=4)$. (C) Cells were transfected with siNT or siVLDLr. After $48 \mathrm{~h}$, cells were pretreated with or without 100ng/L SalA for $30 \mathrm{~min}$, and then subjected to hypoxia for $8 \mathrm{~h}$. The protein levels of Bip, p-PERK, PERK, CHOP, and $\beta$-actin were detected by immunoblot analysis. A representative blot was shown $(\mathrm{n}=3)$.

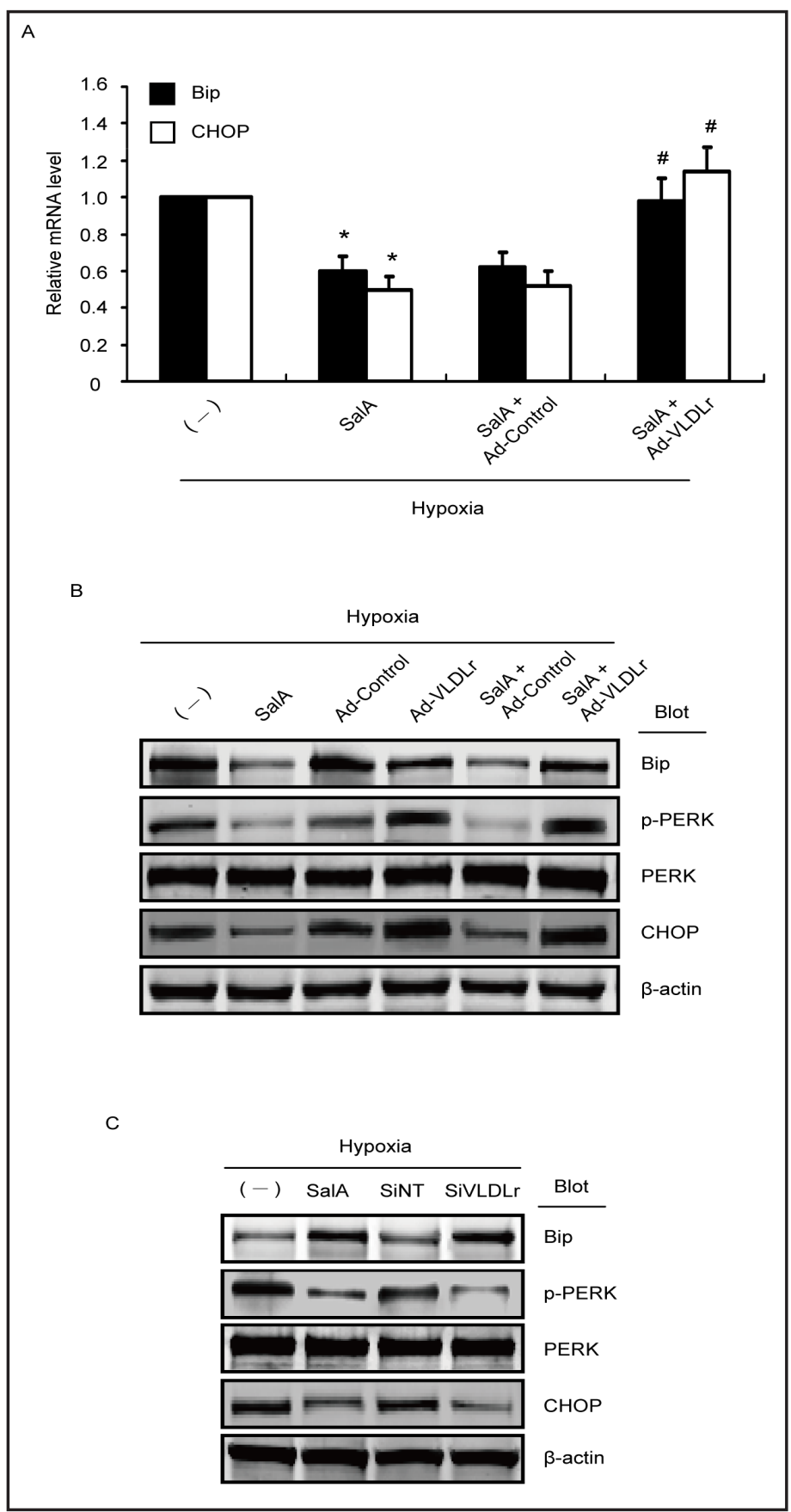

biology processes of folding of secretory and membrane proteins, calcium homeostasis, and lipid biosynthesis were implemented in ER lumen. The impaired ER homeostasis results in accumulation of unfolded or misfolded proteins in the ER, thus directing to the activation of a special cellular process called UPR, which oftentimes represents the event of ER stress [19]. Primarily, the goal of UPR is to alleviate ER stress and maintain cell survival. If ER stress is prolonged or too severe, signalling switches from pro-survival to pro-death, thus triggering ER stress-induced apoptosis [20,21]. More recently, compelling evidences indicated the role of ER stress and UPR in the vascular complications [22]. Endothelial cells, always exposed to multi-factor and multi-event status in blood, are very dynamic, metabolically active cells, with a high volume of protein synthesis, which predispose them to ER stress [23]. Damage of endothelial cells can result not only from oxidative stress, but also from conditions of ER

\section{KARGER}


stress [24]. In our study, hypoxia undoubtedly led to endothelial ER stress and apoptosis (Fig. 1 and Fig. 2).

SalA is one of the main active, water-soluble ingredient in S. miltiorrhiza, which is commonly named "Danshen" in China, and has been widely used as a medicinal plant for more than two thousand years [9]. Nowadays, modern medical research described many beneficial effects of SalA on cardiovascular system [10]. SalA was suggested to be effective in anti-oxidation, anti-apoptosis, and anti-inflammation in numerous pathological stimuliinduced cardiovascular injury [10]. In the present study, we showed that SalA protected endothelial cells from ER stress and apoptosis (Fig. 1 and Fig. 2), a finding that further favor the beneficial effect of SalA on vasculature system. The mechanisms elucidating the role of SalA in regulation of biological processes in endothelial cells, vascular smooth muscle cells, and cardiomyocytes have been investigated [25-27]. It has been described that SalA might be a cardiovascular protective agent during reperfusion injury, mainly through its antiapoptosis activity via induction of Bcl-2 and inhibition of Bax [28, 29]. In agreement with these data, we showed that SalA abolished suppression of Bcle-2 and enhancement of Bax induced by hypoxia (Fig. 1B).

VLDLr is found throughout the body, with particularly high expression in fatty acid tissues due to their high level of triglycerides, VLDLr's primary ligand. These tissues include those of the heart, skeletal muscle, and adipose layer. In addition, the receptor is also found in endothelial cells $[30,31]$. As a peripheral remnant lipoprotein receiver, VLDLr is commonly linked to the lipid metabolism. More recently, its biological functions extended to include signal transduction, cellular proliferation, and tumor pathogenesis [4]. With regard to endothelial cells, there were some interesting findings. VLDLr, as a novel endothelial cell receptor for fibrin, promoted fibrin-dependent leukocyte transmigration and thereby inflammation [7]. The suppression of endothelial cell proliferation by tissue factor pathway inhibitor was mediated by VLDLr [25]. Loss of VLDLr activated retinal vascular endothelial cells and promoted angiogenesis [6]. Further, it was reported that VLDLr was essential for the inhibition of cell division in microvascular endothelial cells [8]. Therefore, all these evidences proposed a crucial action of VLDLr involved in endothelial biology. In our study, SalA effectively suppressed VLDLr expression (Fig. 3). Meanwhile, overexpression of VLDLr abolished the protective role of SalA in the endothelial ER stress (Fig. 5) and apoptosis (Fig. 6). These results indicated that VLDLr be a mediator involved in ER stress and apoptosis, and SalA may be a novel antagonist against VLDLr and its related actions. However, the intensity of endothelial VLDLr expression was varied in different vessel sites, which was detected abundantly in the endothelium of capillaries and small arterioles, in contrast, little or low amount in aortas, veins or venules [31]. Therefore, SalA might be beneficial for these endothelial cells that express lower amount of VLDLr, because it is difficult for SalA to suppress the effects induced by highly expressed VLDLr (Fig. 5B and 6B).

The hypoxia-induced enhancement in VLDLr expression is more likely a general event, as hypoxia has previously been suggested to promote $v l d l r$ mRNA expression in Müller cells and human monocytes [32,33]. Our previous study indicated that HIF-1 $\alpha$ triggered increase in VLDLr expression [17]. However, in the present study, SalA pretreatment did not result in obvious alteration of HIF- $1 \alpha$ protein expression compared with control group (Fig. 4A). Interestingly, SalA inhibited vldlr gene promoter activity (Fig. 4C). Furthermore, hypoxiainduced recruitment of HIF-1 $\alpha$ to the vldlr gene promoter was hampered markedly by SalA pretreatment (Fig. 4C). Collectively, we concluded that SalA might inhibit HIF-1 $\alpha$ binding to $v l d l r$ gene promoter, thus leading to suppression of VLDLr expression.

In conclusion, our results demonstrate, for the first time, that SalA represses hypoxiainduced endothelial ER stress and apoptosis in HUVECs via inhibited binding of HIF-1 $\alpha$ to $v l d l r$ gene promoter and thus suppressed VLDLr expression. Elucidation of the molecular mechanisms by which SalA protects endothelial cells from ER stress and apoptosis may have significance for therapy to reduce hypoxia-induced vascular injury. 
Xie et al.: SalA Attenuates Hypoxia-Induced Endothelial ER Stress

\section{Acknowledgements}

This work was supported by Natural Science Foundation of Shanghai Municipal Science and Technology Commission (13ZR1437300, 14ZR1437400) and outstanding youth medical talents of Pudong Health Bureau of Shanghai (PWRq2013-10). We are grateful to Dr. Dan Yang for critical reading of the manuscript.

\section{References}

1 Wu CX, Liu R, Gao M, Zhao G, Wu S: Pinocembrin protects brain against ischemia/reperfusion injury by attenuating endoplasmic reticulum stress induced apoptosis. Neurosci Lett 2013;546:57-62.

2 Salminen A, Kauppinen A, Hyttinen JM, Toropainen E, Kaarniranta K: Endoplasmic reticulum stress in agerelated macular degeneration: trigger for neovascularization. Mol Med 2010;16:535-542.

3 Zhou AX, Tabas I: The UPR in atherosclerosis. Semin Immunopathol 2013;35:321-332.

4 Takahashi S, Sakai J, Fujino T, Hattori H, Zenimaru Y: The very low-density lipoprotein (VLDL) receptor: characterization and functions as a peripheral lipoprotein receptor. J Atheroscler Thromb 2004;11:200208.

5 Xia CH, Lu E, Liu H, Du X, Beutler B: The role of Vldlr in intraretinal angiogenesis in mice. Invest Ophthalmol Vis Sci 2011;52:6572-6579.

-6 Jiang A, Hu W, Meng H, Gao H, Qiao X: Loss of VLDL receptor activates retinal vascular endothelial cells and promotes angiogenesis. Invest Ophthalmol Vis Sci 2009;50:844-850.

7 Yakovlev S, Mikhailenko I, Cao C, Zhang L, Strickland DK: Identification of VLDLR as a novel endothelial cell receptor for fibrin that modulates fibrin-dependent transendothelial migration of leukocytes. Blood 2012;119:637-644.

8 Oganesian A, Armstrong LC, Migliorini MM, Strickland DK, Bornstein P: Thrombospondins use the VLDL receptor and a nonapoptotic pathway to inhibit cell division in microvascular endothelial cells. Mol Biol Cell 2008;19:563-571.

-9 Wang L, Zhou GB, Liu P, Song JH, Liang Y, Yan XJ, Xu F, Wang BS, Mao JH, Shen ZX, Chen SJ, Chen Z: Dissection of mechanisms of chinese medicinal formula realgar-indigo naturalis as an effective treatment for promyelocytic leukemia. Proc Natl Acad Sci USA 2008;105:4826-4831.

10 Ho JH, Hong CY: Salvianolic acids: Small compounds with multiple mechanisms for cardiovascular protection. J Biomed Sci 2011;18:30.

11 Gan Y, Shen YH, Wang J, Wang X, Utama B: Role of histone deacetylation in cell-specific expression of endothelial nitric-oxide synthase. J Biol Chem 2005;280:16467-16475.

12 Yang D, Xie P, Liu Z: Ischemia/Reperfusion-Induced MKP-3 Impairs Endothelial NO Formation via Inactivation of ERK1/2 Pathway. PLoS ONE 2012;7:e42076.

13 Hempel SL, Haycraft DL, Hoak JC, Spector AA: Reduced prostacyclin formation after reoxygenation of anoxic endothelium. Am. J. Physiol 1990;259:C738-C745.

14 Yang D, Guo S, Zhang T, Li H: Hypothermia attenuates ischemia/reperfusion-induced endothelial cell apoptosis via alterations in apoptotic pathways and JNK signaling. FEBS Lett 2009;583:2500-2506.

15 Basha B, Samuel SM, Triggle CR, Ding H: Endothelial dysfunction in diabetes mellitus: possible involvement of endoplasmic reticulum stress? Exp Diabetes Res 2012:481840.

16 Yang D, Zhang P, Wang T, Gao L, Qiao Z, Liang Y, Yu B: SalA attenuates ischemia/reperfusion-induced endothelial barrier dysfunction via down-regulation of VLDL receptor expression. Cell Physiol Biochem 2014;33:747-757.

17 Yang D, Gao L, Wang T, Qiao Z, Liang Y, Zhang P: Hypoxia triggers endothelial endoplasmic reticulum stress and apoptosis via induction of VLDL receptor. FEBS Lett 2014;10.1016/j.febslet.2014.09.046.

18 Yoshida H: ER stress response, peroxisome proliferation, mitochondrial unfolded protein response and Golgi stress response. IUBMB Life 2009;61:871-879.

19 Kanekura K, Suzuki H, Aiso S, Matsuoka M: ER stress and unfolded protein response in amyotrophic lateral sclerosis. Mol Neurobiol 2009;39:81-89. 
Xie et al.: SalA Attenuates Hypoxia-Induced Endothelial ER Stress

20 Urra H, Dufey E, Lisbona F, Rojas-Rivera D, Hetz C: When ER stress reaches a dead end. Biochim Biophys Acta 2013;1833:3507-3517.

21 Sano R, Reed JC: ER stress-induced cell death mechanisms. Biochim Biophys Acta 2013;1833:3460-3470.

22 Minamino T, Kitakaze M: ER stress in cardiovascular disease. J Mol Cell Cardiol 2010;48:1105-1110.

-23 Witte I, Horke S: Assessment of endoplasmic reticulum stress and the unfolded protein response in endothelial cells. Methods Enzymol 2011;489:127-146.

-24 Scull CM, Tabas I: Mechanisms of ER stress-induced apoptosis in atherosclerosis. Arterioscler Thromb Vasc Biol 2011;31:2792-2797.

25 Yang XY, Qiang GF, Zhang L, Zhu XM, Wang SB, Sun L, Yang HG, Du GH: Salvianolic acid a protects against vascular endothelial dysfunction in high-fat diet fed and streptozotocin-induced diabetic rats. J Asian Nat Prod Res 2011;13:884-894.

26 Zhang YQ Tang Y, Wu AL, Zhu HB: Salvianolic acid a displays cardioprotective effects in in vitro models of heart hypoxia/reoxygenation injury. J Asian Nat Prod Res 2010;12:899-915.

27 Oh KS, Oh BK, Mun J, Seo HW, Lee BH: Salvianolic acid a suppress lipopolysaccharide-induced nf-kappab signaling pathway by targeting ikkbeta. Int Immunopharmacol 2011;11:1901-1906.

28 Pan H, Li D, Fang F, Chen D, Qi L, Zhang R, Xu T, Sun H: Salvianolic acid a demonstrates cardioprotective effects in rat hearts and cardiomyocytes after ischemia/reperfusion injury. J Cardiovasc Pharmacol 2011;58:535-542.

-29 Fan H, Yang L, Fu F, Xu H, Meng Q Zhu H, Teng L, Yang M, Zhang L, Zhang Z, Liu K: Cardioprotective effects of salvianolic acid a on myocardial ischemia-reperfusion injury in vivo and in vitro. Evid Based Complement Alternat Med 2012;2012:508938.

-30 Lv P, Miao SB, Shu YN, Dong LH, Liu G, Xie XL, Gao M, Wang YC, Yin YJ, Wang XJ, Han M: Phosphorylation of smooth muscle 22alpha facilitates angiotensin ii-induced ros production via activation of the pkcdeltap47phox axis through release of pkcdelta and actin dynamics and is associated with hypertrophy and hyperplasia of vascular smooth muscle cells in vitro and in vivo. Circ Res 2012;111:697-707.

-31 Wyne KL, Pathak K, Seabra MC, Hobbs HH: Expression of the vldl receptor in endothelial cells. Arterioscler Thromb Vasc Biol 1996;16:407-415.

32 Loewen N, Chen J, Dudley VJ, Sarthy VP, Mathura JR Jr: Genomic response of hypoxic muller cells involves the ver11y low density lipoprotein receptor as part of an angiogenic network. Exp Eye Res 2009;88:928937.

33 Nakazato K, Ishibashi T, Nagata K, Seino Y, Wada Y, Sakamoto T, Matsuoka R, Teramoto T, Sekimata M, Homma Y, Maruyama Y: Expression of very low density lipoprotein receptor mrna in circulating human monocytes: Its up-regulation by hypoxia. Atherosclerosis 2001;155:439-444. 